\title{
Statistical Analysis in Complex-Valued Wavelet Analysis of Voltage-Sensitive Dye Mapping
}

\author{
J Bardonova $^{1}$, I Provaznik ${ }^{1}$, M Novakova $^{2}$, J Sekora $^{1}$, M Svrcek ${ }^{1}$ \\ ${ }^{1}$ Brno University of Technology, Brno, Czech Republic \\ ${ }^{2}$ Masaryk University Brno, Brno, Czech Republic
}

\begin{abstract}
The aim of this study is to learn more about influence of the voltage sensitive dye to the tissue during optical mapping experiments on isolated animal hearts. There were recordings from all phases of the experiment examined to exclude possible negative influence of the dye to the tissue.

Consecutive individual heart cycles extracted from ECG signals were transformed to time-frequency domain and divided into parts corresponding to $P-Q$ segments, $Q R S$ complexes, and ST-T segments in each experimental period. Changes in ECG cycles caused by voltage sensitive dye application were studied during experimental phases. The statistical analysis were performed in time-frequency domain and compared.
\end{abstract}

\section{Introduction}

At present, the optical mapping is widely used in cardiac electrophysiology animal experiments $[1,2]$. The principle of optical mapping is an application of voltagesensitive dye (VSD) to examined tissue where it binds to a membrane of cardiac cells. It is necessary to examine recordings from all phases of the experiment to exclude possible negative influence of the dye to the tissue $[3,4]$. Also, the same procedure could be used to detect electrophysiological changes e.g. during ischemia studies.

The continuous wavelet transform (CWT) has been used to detect expected changes. The use of CWT may help to track the changes simultaneously in time and frequency domain. Statistical analysis of CWT results supports hypothesed changes.

\section{Methods}

In this study, influence of the voltage sensitive dye to the tissue during optical mapping experiments on isolated animal hearts were studied. There were recordings from all phases of the experiment examined to exclude possible negative influence of the dye to the tissue.

\subsection{Experimental setup and protocol}

15 rabbit hearts were included in the study. Each heart was mounted on a Langendorf apparatus, filled with Krebs-Henseleit $(\mathrm{K}-\mathrm{H})$ solution $(1.25 \mathrm{mM} \mathrm{Ca} 2+)$ and placed in a bath $\left(37^{\circ} \mathrm{C}\right)$. The hearts were perfused at the constant pressure of $85 \mathrm{mmHg}$. The hearts were stabilized for 30 minutes. After control period, the hearts were perfused with $1 \mathrm{mM}$ solution of VSD di-4-ANNEPS diluted in K-H solution, duration of loading period was 20-25 minutes. Than dye was washed out for next 20-25 minutes.

ECG signals were continuously recorded during whole experiment. Than long term trends were studied from ECG signals via analysis of 15 minutes of loading period, which means period of VSD application, and 15 minutes of wash-out. ECG from both periods were compared with ECG from control period.

Recording system was assembled with three touchless orthogonal lead system with $\mathrm{Ag}-\mathrm{AgCl}$ disc electrodes. The signals were digitized by a 12-bit AD converter at 2 $\mathrm{kHz}$ sampling rate using a data acquisition multifunction card PCI-6111E (National Instruments, USA). The digital signals were stored on a hard disk for further off-line processing.

\subsection{Analysed data}

10 -second segments at the beginning of each minute of records were extracted and used. In all phases, control period, loading of VSD and washout period, heart cycles were detected. For each minute of recordings, median heart cycle was determined and divided into P-Q intervals, QRS complexes, and S-T intervals (see Figure 1). Median ECG cycles are transformed by continuous wavelet transform. 


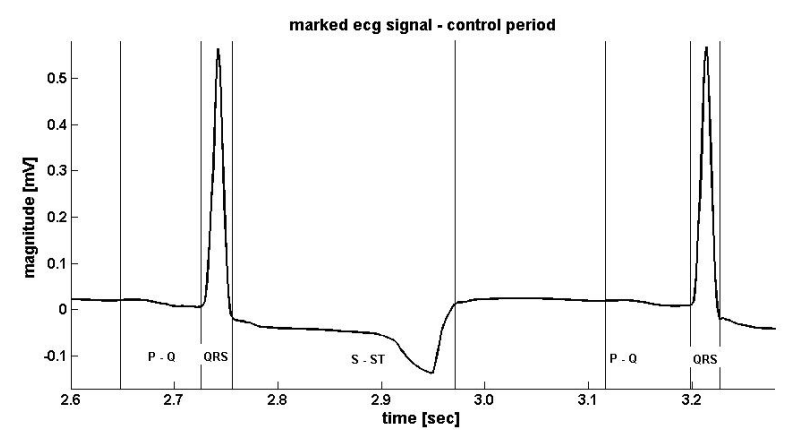

Figure 1. ECG signal during control period with marked P-Q segment, QRS complex and ST-T segment.

\subsection{Wavelet transform}

Continuous wavelet transform (CWT) is defined as correlation of a signal $\mathrm{x}(\mathrm{t})$ with wavelets $\mathrm{g} *[(\mathrm{t}-\tau) / \lambda]$, where $\tau$ is time shift, $\lambda$ is time dilation, and $*$ represents complex conjugate.

$$
C W T(\lambda, \tau)=\int_{-\infty}^{\infty} \frac{1}{\sqrt{\lambda}} \mathrm{g} *\left(\frac{\mathrm{t}-\tau}{\lambda}\right) \mathrm{x}(\mathrm{t}) \mathrm{dt}
$$

Median heart cycles were transformed by CWT using complex Gaussian wavelet No.2 which was experimentally chosen for its good time-frequency resolution ratio. Time-frequency images were divided into parts corresponding to P-Q segment, QRS complex and ST-T segments as was marked in time domain.

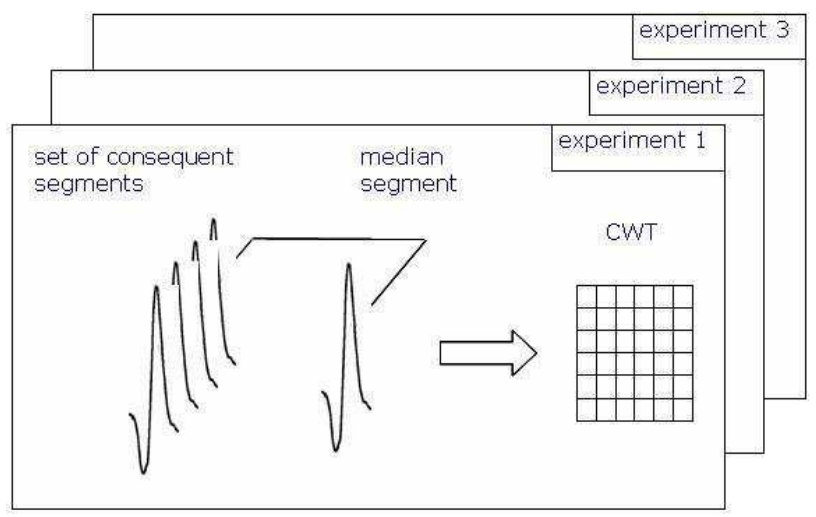

Figure 2. Schematic diagram of preprocessing data for continuous wavelet transform analysis.
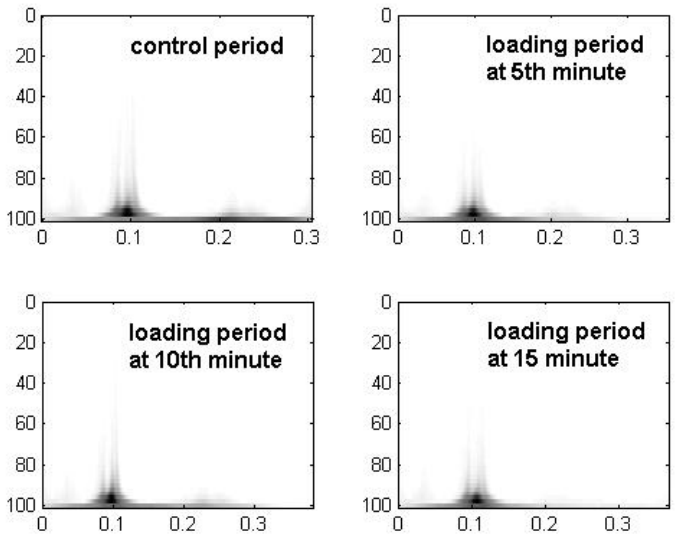

Figure 3. Continuous-time wavelet transform of ECG signals. Magnitude spectrum of median ECG cycle in (a) control period, (b) loading period at 5th minute, (c) loading period at 10th minute, (d) loading period at 15 th minute. Vertical axis: scales $\lambda$ from 3 to 60 (corresponds to frequencies $5 \mathrm{~Hz}$ to $150 \mathrm{~Hz}$ ).

\subsection{Statistical analysis of wavelet images}

Differences of magnitude spectra should be confirmed by statistical test. Significant abnormalities were assessed by comparing the mean value of each of CWT of the two studied populations by means of a two-way two-tailed ttest to test for the null hypothesis that means are equal.

First population was build from ECG segments in control period, second populations from ECG segments in all other periods.

Time-scale images of recordings from control period were compared with time-scale images of recordings from each minute (1 to 15 ) of loading period and each minute (1 to 15 ) of washout period.

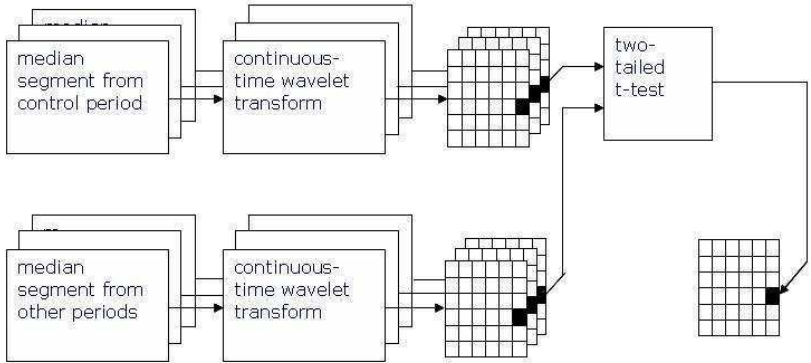

Figure 4. Schematic representation of the time-frequency $\mathrm{t}$-test analysis method generating $\mathrm{p}$-value maps.

First population of data for t-test were composed from corresponding time-frequency components from timefrequency spectra of all experiments in control period (black squares in Figure 4). Second population of data were composed from time-frequency spectra of all 
experiments in other periods. t-test was performed on the described two populations of data. Thus, p-value maps with horizontal and vertical axes corresponding to timefrequency images were generated. Each component is result from t-test.

Figure 5 represents data to be t-test analysed and the result of the analysis. The left upper panel shows magnitude time-frequency spectrum of whole median ECG cycle from control period of one experiment. Similarly, the spectrum generated from 5th minute of loading period is shown in upper right panel. Here, P-Q interval is marked by two vertical lines in both panels. Data populations for t-test are composed from the marked intervals across all experiments and statistically tested. The lower panel represents the results of t-test coded in gray scale (only P-Q interval is shown here). Dark areas of the image correspond to $p<0.05$. The darker area means the more rejected is the null hypothesis.

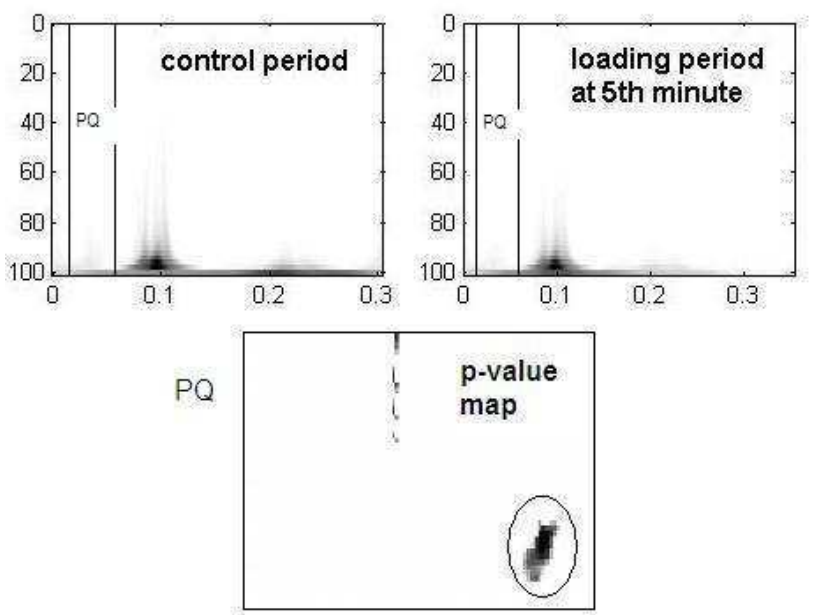

Figure 5. Changes in magnitude spectra confirmed by ttest. (a) magnitude spectrum of ECG cycle during control period, (b) magnitude spectrum of ECG cycle during each minute loading and washout periods, (c) p-value map correspond to PQ segments differences in timefrequency domain.

\section{Results}

In this study, $p$-value maps for time-frequency spectra of P-Q segments, QRS complexes and ST-T segments were calculated based on 15 experiments. Analysis of each mentioned segment was done minute-by-minute for 15 minutes of loading period and 15 minutes of washout period. Thus, sequences of $30 \mathrm{p}$-value maps for each ECG cycle segment were generated.

Figure 6 represents an example of the analysis result for first 6 minutes of loading period for P-Q segment. Here one can see persistent significant changes in the lower right part of the p-value maps. It suggests electrophysiological changes expressing in the end of P$\mathrm{Q}$ interval in lower frequencies. Such changes were proved during whole loading period and whole washout period with fading trend in the end of washout. There were no changes in $8^{\text {th }}$ minute and later. The analysis method fails from time to time (see 3 rd and 4 th minute in Figure 4).

The results are randomly disturbed with characteristic patterns of vertical lines (see upper parts of panels in Figure 4). The lines are usually one sample of width. It was revealed as an combined influence of high frequency noise and imprecise detection of beginning of intervals.

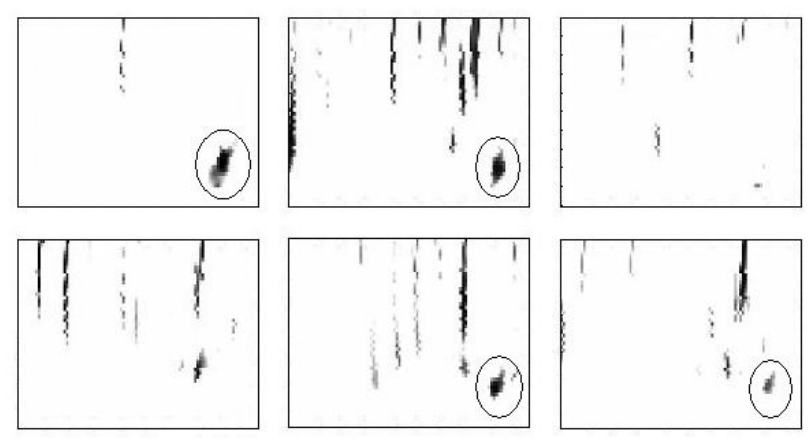

Figure 6. p-value maps for P-Q segment for first 6 minutes during loading period. Significant changes are marked with ellipses.

Although minor but persistent changes were expected in QRS complex analysis, only insignificant spots were detected in p-value maps. Two or three independent changes represented by relatively large dark areas in the maps can be found within whole 15 minutes of loading period. However, these changes are independent from each other, they begin and end in various parts of the loading period in various parts of the p-value maps. Thus, changes are proved but it is not possible to relate them to known electrophysiological activities of the heart without further analysis. Similar results can be found for QRS complex analysis of washout period of the experiments.

In ST-T segment analysis, the results are largely superimposed with highly random disturbations due to noise and imprecise of detection of S wave. No persistent large electrophysiological change during loading period proved by $\mathrm{t}$-test for $\mathrm{p}<0.05$ can be found. The same findings can be described for washout period.

\section{Discussion and conclusions}

Time-frequency domain statistical analysis using magnitude of CWT revealed large changes in P-Q segment during VSD loading, minor changes in QRS complex and almost no shape changes in ST-T segment. Changes during P-Q segment were restored in wash-out. 
Minor QRS changes were also restored in wash-out. These findings correspond to our previous studies based on time-domain.

t-test analysis was applied to time-frequency images of ECG signals. Significant changes caused by application of voltage-sensitive dyes were searched for. ECG signals were analysed segment by segment (P-Q, QRS, ST-T) during loading and washout period. The segments were transformed to time-frequency domain. Significant changes were found in time-frequency morphology of P$\mathrm{Q}$ segment just after beginning of loading. These changes disappeared with beginning of washout. Changes in another part of time-frequency domain appeared at the same time and disappeared during washout. Analysis of QRS complexes and ST-T segments did not prove any significant change between the two periods. Physiological background of time-frequency changes is under investigation now in order to explain why different parts of segments are affected.

\section{Acknowledgements}

The research was supported by the grants 102/07/P521 and 102/07/1473 from GACR.

\section{References}

[1] Inagaki M, Hidaka I, Aiba T, Tatewaki T, Sunagawa K, Sugimachi M. High Resolution Optical Mapping of Cardiac Action Potentials in Freely Beating Rabbit Hearts. Proceedings of the 26th Annual International Conference of the IEEE EMBS San Francisco; CA, USA September 15,2004
[2] Salama G. Optical Mapping: Background nad Historical Perspective. In Pptical Mapping of Cardiac Excitation and arrythmias. Rosenbaum D, Jelife J, Ed. Armonk, NY. Futura Publishing, Inc.., Ch 1., 2001

[3] Qin H, Kay MW, Chattipakorn N, Redden DT, Ideker RE, Rogers JM. Effects of Heart Isolation, Voltage-Sensitive Dye, and Electromechanical Uncoupling agents on Ventricular Fibrillation. Am J Physiol Heart Circ Physiol 284: H1818-H1826, 2003

[4] Novakova M, Blaha M, Bardonova J, Provaznik I. Comparison of the Tissue Response during the Loading with Voltage-Sensitive Dye in Two Animal Models. Computers in Cardiology 2005;32:535-538, 2005

[5] de Luna AB, Stern S. Future of Noninvasive Electrocardiology. In: Zareba W, Maison-Blanche P, Locati E H. Noninvasive Electrocardiology in Clinical Practice, Futura Publishing Co., 475-478, 2001

Address for correspondence

Jana Bardonova

Department of Biomedical Engineering

Brno University of Technology Brno

Kolejni 4

61200 Brno, Czech Republic

bardona@feec.vutbr.cz 